\title{
EchoGéo
}

15 | 2011

La Belgique, État prospère en décomposition au cœur de l'Europe du nord-ouest

\section{La Belgique, État prospère en décomposition au coeur de l'Europe du nord-ouest}

Introduction

\section{Christian Vandermotten}

\section{(2) OpenEdition}

Journals

\section{Édition électronique}

URL : https://journals.openedition.org/echogeo/12320

DOI : $10.4000 /$ echogeo. 12320

ISSN : 1963-1197

Éditeur

Pôle de recherche pour l'organisation et la diffusion de l'information géographique (CNRS UMR 8586)

Référence électronique

Christian Vandermotten, « La Belgique, État prospère en décomposition au coeur de l'Europe du nordouest », EchoGéo [En ligne], 15 | 2011, mis en ligne le 11 avril 2011, consulté le 03 août 2021. URL http://journals.openedition.org/echogeo/12320 ; DOI : https://doi.org/10.4000/echogeo.12320

Ce document a été généré automatiquement le 3 août 2021.

EchoGéo est mis à disposition selon les termes de la licence Creative Commons Attribution - Pas d'Utilisation Commerciale - Pas de Modification 4.0 International (CC BY-NC-ND) 


\title{
La Belgique, État prospère en décomposition au coeur de l'Europe du nord-ouest
}

\author{
Introduction
}

\author{
Christian Vandermotten
}

1 La Belgique a 9 millions d'habitants. Son contrôle sur des territoires d'outre-mer particulièrement riches, une batellerie importante, comme en atteste la photo d'un navire marchand à quai devant la ville d'Anvers, lui permet de tenir dans le monde une place de grande puissance économique et nourrit un commerce extérieur important. La Belgique importe des denrées alimentaires, des matières premières pour son industrie, du pétrole, des automobiles et exporte des produits industriels (aciers, machines, ciment, lin, tissus) et des articles de consommation (endives, raisins, etc.).

2 Dans ce pays divisé entre partie de langue germanique au nord, wallonne au sud, la population de la capitale, en partie d'origine flamande, adopte de plus en plus la langue française dont le rayonnement est plus grand. Mais l'antagonisme entre les deux éléments de la population belge, flamand et wallon, peut encore mettre en péril l'unité de l'Etat.

3 Le pays flamand est très peuplé, à part l'infertile Campine, car la natalité y reste forte. Les paysans n'y disposent que de très petites exploitations, avec le beurre, les bêtes à viande, les produits de basse-cour, le lin, le tabac, des légumes, du houblon, de la chicorée, des produits obtenus en serre, comme les fleurs ou, autour de Bruxelles, les endives et les raisins. Des ouvriers flamands vont même travailler dans le nord de la France. La majorité des Flamands habite la campagne et de nombreuses petites villes. Les usines, qui trouvent partout des ouvriers et des ouvrières, peuvent s'y disperser, d'autant qu'elles transforment des matières légères, textiles surtout. Mais les ports maritimes transforment de plus en plus les matières premières importées et les charbonnages de Campine et les rives du canal Albert attirent l'industrie lourde.

4 Au contraire, la population wallonne se groupe surtout dans les centres industriels, sur les pays noirs du bassin houiller du sud, qui a fondé la spécialisation dans l'industrie lourde, sauf à Verviers, spécialisée dans le travail de la laine. La natalité wallonne est faible et les pays ruraux de l'Ardenne se dépeuplent. 
Dans cette vieille Belgique commerçante, depuis le Moyen âge, les capitaux accumulés ont permis le développement industriel moderne. Bruxelles est une importante place financière où sont implantées des entreprises qui ont des intérêts dans le monde entier, en particulier au Congo belge. Anvers se classe, avec Rotterdam, en tête des ports européens, mais chaque projet visant à améliorer les liaisons entre Anvers et le Rhin se heure à l'hostilité néerlandaise. Les Belges, en creusant le canal Albert et en se refusant à transformer le cours de la Meuse à la frontière des Pays-Bas, limitent l'extension du trafic néerlandais vers les pays de la Meuse. Néanmoins, depuis 1945, la Belgique et les Pays-Bas s'efforcent de créer le Bénélux, même si les rivalités commerciales en retardent la réalisation.

Que reste-t-il de cette présentation de la Belgique voici un demi-siècle $?^{1}$ Les textes publiés dans cette livraison d'EchoGéo ne répondent évidemment pas à tous les aspects de cette question, mais en présentent certains. Ils pourront sans doute contribuer à la déconstruction d'une représentation qui prévaut sans doute encore pour une part, après ce demi-siècle, parmi une grande partie du public, complétée par une incompréhension - en particulier auprès d'un public français habitué aux rationalités jacobines - face à un processus de fédéralisation et de décomposition politique couplé à du pragmatisme au quotidien.

7 La Belgique a 10,8 millions d'habitants, en croissance modeste mais qui se poursuit malgré une fécondité réduite depuis le début des années soixante-dix à moins de 2 enfants par femme. Les prévisions sont de 12,4 millions d'habitants en 2050, toujours avec moins de 1,8 enfants par femme. Mais la Flandre est maintenant moins féconde que la Wallonie; elle ne va plus à la messe, même si les piliers de l'encadrement catholique (écoles, mutuelles, syndicats) y gardent toute leur puissance. Elle présente un curieux mélange de repli identitaire, de vote à droite et d'ouverture sociétale, de modernité économique et de méfiance envers la grande ville et ses élites cosmopolites (voir les contributions de C. Vandermotten et de G. Van Hamme \& G. Pion). Cette Flandre fière de ses réussites économiques reste linguistiquement frileuse, attachée à la défense, voire à la reconquête de son sol, comme lorsqu'elle était économiquement et socialement dominée par la bourgeoisie belge francophone, qui tenait aussi le haut du pavé dans les villes flamandes. Etonnant mélange de post-modernité et d'ancrage dans le terroir, que l'on peut faire remonter pour une part, en ce qui concerne ce dernier, à l'époque où la Contre-réforme catholique a vidé la Flandre de ses élites urbaines passées au protestantisme et qui fut conforté par les conditions économiques et sociales dans lesquelles a émergé le mouvement flamand au XIX ${ }^{e}$ siècle. Situation bien différente en tout cas de celle de la bourgeoisie commerçante et cosmopolite néerlandaise, qui ne nourrit aucun sentiment d'infériorité linguistique et troque volontiers le néerlandais pour l'anglais, affaires obligent.

Quant à la Wallonie, qui voit maintenant la population flamande vieillir plus vite que la sienne, elle n'est plus ni charbonnière, ni même dominée par l'industrie lourde, au point que la part de ses actifs occupés dans l'industrie est devenue moindre qu'en Flandre, qui s'est entre-temps industrialisée massivement durant la période fordiste. Mais les marques sociales et environnementales de son passé industriel subsistent et l'ancien axe charbonnier vote toujours autant socialiste. L'Ardenne et les campagnes en général ont cessé d'y être des zones d'exode rural séculaire ; la rurbanisation y assure presque partout une croissance, parfois sensible, de la population.

$9 \quad$ Et entre les deux, il y a Bruxelles, qui s'est transformée de petite capitale en une ville internationale majeure, largement fréquentée par des travailleurs flamands qui 
s'empressent de quitter chaque jour cette cité que la Flandre revendique comme sa capitale, où elle exige la plus parfaite parité linguistique, même si le néerlandais y est sans doute moins pratiqué que l'anglais, et, à domicile, que l'arabe marocain ou le turc. Mais pas nécessairement pour autant ville aimée en Wallonie francophone, où certains continuent d'y voir la ville de ce capitalisme belge auquel ils attribuent tous les maux qui se sont abattus sur leur économie. Même si Bruxelles innerve le Brabant wallon, dont elle assure le dynamisme périmétropolitain, tout comme la ville de Luxembourg assure largement celui de la province belge homonyme. Mais ce sont là deux provinces dont la sociologie est bien différente de celle des deux provinces wallonnes les plus peuplées, le Hainaut et Liège. En fait, il n'y a plus vraiment de grand capitalisme belge : le capitalisme industriel et celui des vieilles firmes coloniales a disparu, s'est reconverti en capitalisme financier, dissous aujourd'hui dans la finance française et néerlandaise. Il y a de nombreuses implantations de firmes transnationales, en particulier dans les zones industrialo-portuaires et dans les espaces périphériques du Nord-est - les landes infertiles de la Campine n'existent plus que dans des réserves naturelles ou incluses dans des parcs touristiques - ; des petits et moyens entrepreneurs flamands ; trop peu de wallons, comme souvent dans les régions de vieille industrialisation.

L'évolution du commerce extérieur a accompagné les mutations économiques, industrialisation fordiste et désindustrialisation post-fordiste. L'importance du commerce maritime colonial s'est estompée face à l'essor des lignes régulières transportant des conteneurs reliant Anvers avec les États-Unis ou l'Extrême-Orient. Les bateaux, sauf ceux de croisière, n'accostent plus sur les rives de l'Escaut, devant la ville d'Anvers, mais desservent les vastes espaces de stockage au nord de la ville, jusqu'à la frontière néerlandaise, ainsi que les nouveaux bassins de la rive droite du fleuve, ou encore le port de Zeebrugge, dont l'accès à la haute mer est direct. Les navires pétroliers ne s'aventurent plus dans les méandres de l'estuaire de l'Escaut, mais le pétrole brut arrive par oléoduc depuis Rotterdam. Et même si les Pays-Bas mettent parfois encore quelque mauvaise volonté à favoriser les relations d'Anvers avec son hinterland rhénan, en freinant par exemple l'aménagement du «Rhin de fer» ferroviaire, un canal à très grand gabarit n'en relie pas moins directement Anvers au Rhin. Néanmoins, la Belgique n'est pas en reste, puisque le «bouchon de Lanaye », qui limite le gabarit des bateaux passant de Liège à la Basse-Meuse vers les Pays-Bas, n'a pas encore sauté - mais les travaux devraient bientôt commencer.

11 La part du commerce extérieur (importations plus exportations) est passée de $55 \%$ du PIB en 1953 à $174 \%$ aujourd'hui (dont $34 \%$ au lieu de $9 \%$ pour les échanges de services) et il s'agit pour une large part d'échanges internes au système économique intégré européen : les produits échangés avec les pays voisins (y compris la Grande-Bretagne) représentent en $200956 \%$ du commerce extérieur, contre $45 \%$ en 1953, alors que l'Afrique est passée de 10,0\% à 2,6\%. Dans ce contexte d'intégration fonctionnelle des systèmes de production, la Belgique est tout autant importatrice qu'exportatrice de produits de l'industrie automobile, et non plus comme en 1953 seulement importatrice de pièces détachées, voire de voitures toute montées. La part des produits chimiques, des machines et appareils et du matériel de transport dans les exportations de marchandises est passée de $19 \%$ à $47 \%$, alors que le textile régressait de $19 \%$ à moins de $4 \%$. Dans ce système, la position de transit du pays au coeur de l'Europe du nordouest s'est imposée; elle génère un encombrement toujours plus gênant du réseau 
autoroutier, mais aussi la multiplication des implantations logistiques (contribution de M. Strale).

Plus que jamais, l'unité de l'État est en péril, mais « l'antagonisme entre Flamands et Wallons » qu'évoquait déjà le manuel de 1954 n'est pas une donnée immanente ; c'est un produit de l'histoire dont les deux articles de C. Vandermotten d'une part, de G. Van Hamme \& G. Pion de l'autre, illustrent la genèse et les expressions politiques.

Pour le reste, que reste-t-il encore de cette description scolaire des années cinquante ? Une agriculture flamande très intensive, privilégiant l'élevage hors sol, a oublié le houblon, les raisins de serre et le tabac. Des paysages maintenant mités partout, mais surtout en Flandre, parce que les densités de population y sont plus fortes, par une périurbanisation généralisée ; parce que les Belges ont "une brique dans le ventre ", mais cela aussi est le résultat de politiques délibérées, qui remontent au XIX ${ }^{\mathrm{e}}$ siècle, pour laisser en situation semi-rurale une part importante de la population ouvrière, et qui furent renforcées après la Seconde Guerre mondiale dans le cadre de politiques fordistes de stabilisation sociale et d'encouragement à l'accès à la propriété et à l'usage de la voiture individuelle; parce que les régulations dans l'usage des sols sont globalement laxistes dans un pays où l'État est faible et profondément marqué par le libéralisme, qui a encadré dès l'origine son développement économique précoce. D'où aussi, dans ce pays de propriétaires, une faiblesse du logement social, qui pose aujourd'hui problème, en particulier dans les grandes villes et à Bruxelles, confrontée à une croissance démographique inattendue, en raison d'une reprise de l'immigration et de la fécondité supérieure et de la jeunesse des personnes d'origine immigrée (contribution de C. Dessouroux \& A. Romainville). En tout cas, des paysages qui ne peuvent s'interpréter que comme des construits sociaux, qui remodèlent sans cesse les données «naturelles ", qui ne sont en fait que les formes anciennes de socialisation de la nature (article de V. Van Eetvelde \& M. Antrop).

14 Cette faiblesse des régulations et cette périurbanisation intense ont aussi remodelé la carte de la localisation des services aux personnes et des commerces, très nombreux (contribution de J.P. Grimmeau \& B. Wayens). Les centres commerciaux périphériques ont affaibli les centres-villes, d'où les grands magasins traditionnels ont presque disparu.

15 Pas plus que la "brique dans le ventre ", le surréalisme, l'auto-dérision, la faiblesse du sens de l'État ne sont inscrits dans le patrimoine génétique des Belges. Ce sont des produits d'une histoire séculaire ou pluriséculaire, qui tirent pour une part leurs origines des tensions entre les autonomies des villes marchandes et artisanales, obtenues dès le Moyen-âge par une classe sociale nouvelle, la bourgeoisie urbaine, et la recherche d'affirmation d'un pouvoir central qui tentait de s'imposer à des prérogatives locales et à des privilèges bien affirmés. Pouvoir central qui pouvait en outre apparaitre comme étranger, même s'il était tout à fait légitime dans ses filiations héréditaires. Cela, les auteurs du manuel de 1954 le signalaient déjà, en des termes plus vulgarisés, même s'ils ne pouvaient sans doute pas suffisamment en mettre en valeur les implications dans un tel ouvrage. Si l'on ne connait pas cela, on ne peut rien comprendre à la Belgique, dont les difficultés politiques actuelles, voire la survie, qui n'est pas assurée à terme, inquiètent peut-être plus à l'étranger que dans le pays, qui reste pragmatiquement administré, sinon gouverné, et continue à fonctionner (voir aussi l'article de C. Vandermotten, dans EchoGéo, Sur le Vif, 22 novembre 2007). 
Illustration 1 - Carte de localisation

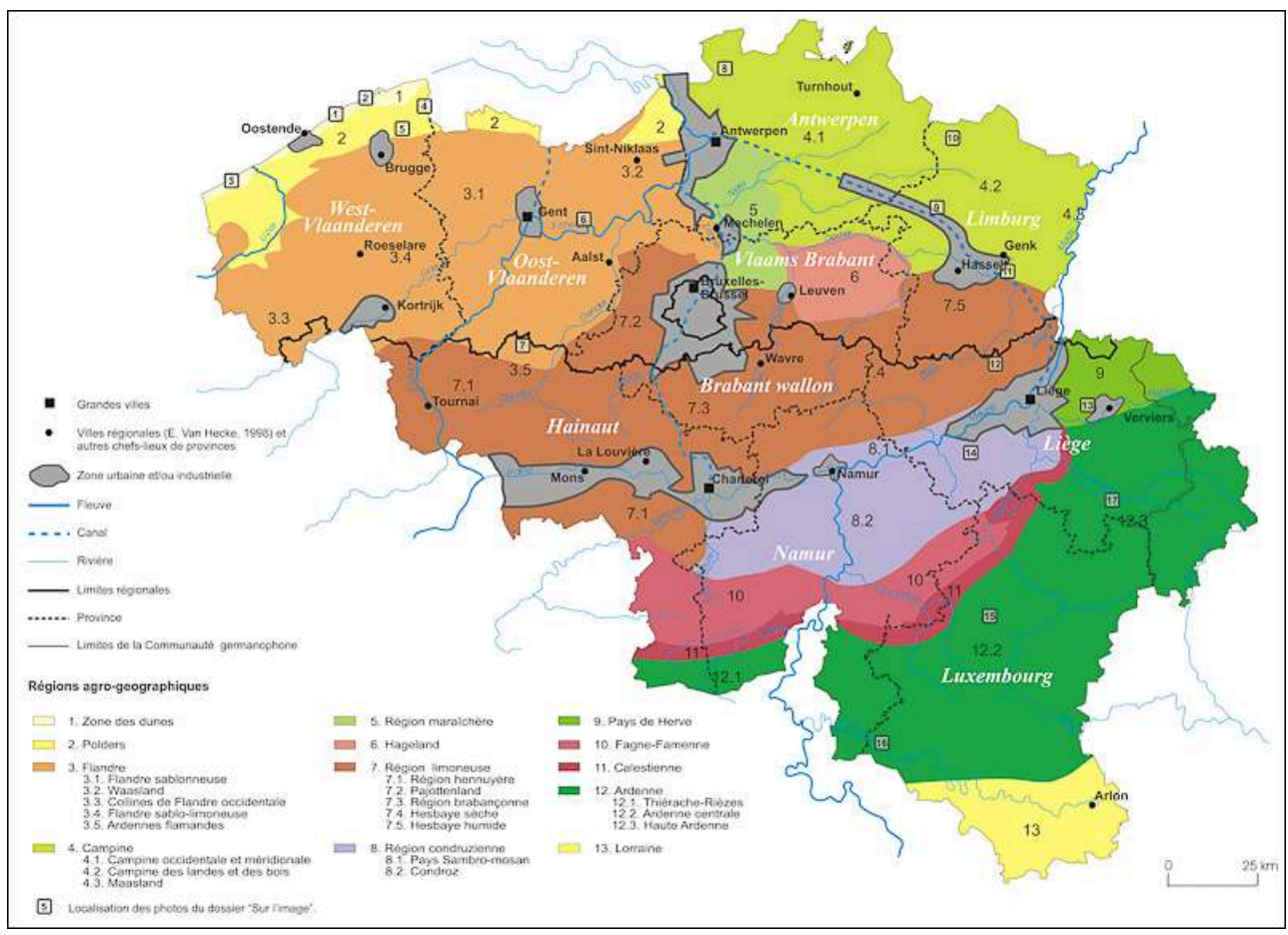

Les numéros dans des carrés renvoient aux photographies présentées dans la rubrique Sur l'Image

\section{NOTES}

1. Lentacker F., Moreau J.P. \& Ozouf M. (3 éd., 1954), Nouveau cours de géographie. Classe de quatrième, Paris, Nathan.

\section{AUTEUR}

\section{CHRISTIAN VANDERMOTTEN}

Christian Vandermotten, Université libre de Belgique, cvdmotte@ulb.ac.be 